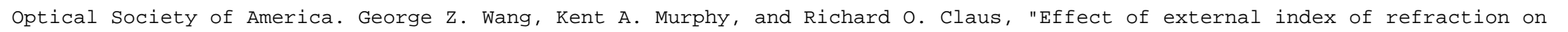

\title{
Effect of external index of refraction on multimode fiber couplers
}

\author{
George Z. Wang, Kent A. Murphy, and Richard O. Claus
}

\begin{abstract}
The dependence of the performance of fused-taper multimode fiber couplers on the refractive index of the material surrounding the taper region has been investigated both theoretically and experimentally. It has been identified that for a $2 \times 2$ multimode fiber coupler there is a range of output-power-coupling ratios for which the effect of the external refractive index is negligible. When the coupler is tapered beyond this region, the performance becomes dependent on the external index of refraction and lossy. To analyze the multimode coupler-loss mechanism, we develop a two-dimensional ray-optics model that incorporates trapped cladding-mode loss and core-mode loss through frustrated total internal reflection.

Computer-simulation results support the experimental observations. Related issues such as coupler fabrication and packaging are also discussed. ㄷ 1995 Optical Society of America
\end{abstract}

\section{Introduction}

For more than 15 years, many of the multimode optical fiber couplers used in communication systems have been fabricated with the fused biconical taper method.1-4 Manufacturing a fused biconical taper coupler involves bringing stripped fibers together, fusing them by applying heat, and stretching them at the same time. This is not only a relatively simple and cost-effective process but al so permits the production of environmentally stable devices. The powercoupling principle for multimode fiber couplers has been studied elsewhere. ${ }^{5-9}$ Even though the dependence of coupler performance on the refractive index of the material external to the tapered region has been investigated extensively for single-mode couplers, ${ }^{10-14}$ little attention regarding this effect has been given to multimode fiber couplers, but it is equally important to coupler design, packaging, and environmental stability.

In this paper we investigate the effect of the external index of refraction on the performance of multimode $2 \times 2$ fiber couplers fabricated with the fused biconical taper technique. The results of this study are intended to hel p understand the loss mecha-

The authors are with the Bradley Department of Electrical Engineering, Fiber \& Electro-Optics Research Center, Virginia Polytechnic Institute and State University, Blacksburg, Virginia 24061-0111.

Received 1 March 1995; revised manuscript received 25 August 1995.

0003-6935/95/368289-05\$06.00/0.

(1) 1995 Optical Society of America. nisms of multimode couplers and to develop low-loss and environmentally stable couplers.

\section{Analysis}

The loss mechanism of a multimode coupler is similar to that of a single tapered multimode fiber, which supports a large number of modes. For a particular guided mode, the effective index satisfies

$$
\mathrm{n}_{\text {clad }}<\beta_{\mathrm{i}} / \mathrm{k}<\mathrm{n}_{\text {core }}
$$

where $\beta_{i}$ is the propagation constant and $k=2 \pi / \lambda$. The modes whose effective indices are close to $n_{\text {clad }}$ are the higher-order modes in reference to the lowerorder modes whose effective indices are close to $\mathrm{n}_{\text {core. }}$ The electrical fields of the higher-order modes extend more into the fiber cladding region than the fields of the lower-order modes. In the tapering process, the effective index of a higher-order mode approaches $\mathrm{n}_{\mathrm{clad}}$, and when they are equal, this mode becomes cut off and is radiated, contributing to loss.

Unlike a single-mode fiber coupler, the core area of a multimode fiber is larger than or at least comparable to that of the cladding area. Because a large number of modes are involved, it would be a laborious task to analyze a multimode coupler with modal theory. Therefore we propose a ray-optics model to calculate somewhat quantitatively the effect of the external index on multimode-fiber-coupler performance. In this model, both the surrounding (external) index of refraction and the cladding thickness are considered. For simplicity, we use a two-dimensional waveguide structure shown in Fig. 1 . The 


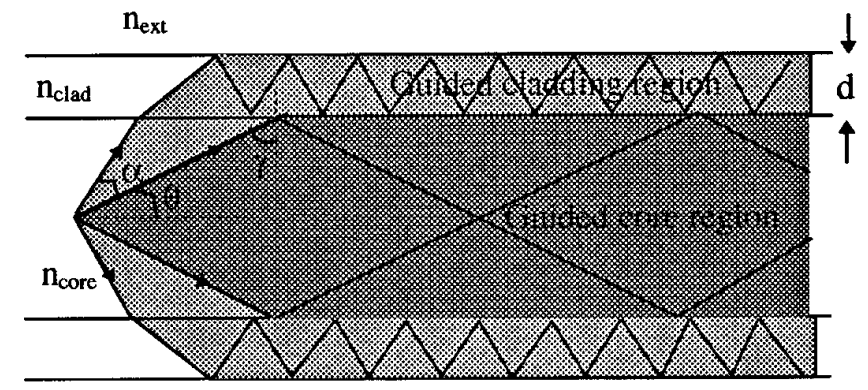

Fig. 1. Multimode fiber taper waveguide.

core, cladding, and external indices of refraction are $\mathrm{n}_{\text {core }}, \mathrm{n}_{\text {clad, }}$ and $\mathrm{n}_{\text {ext }}$, respectively. Normally, $\mathrm{n}_{\text {core }}>$ $\mathrm{n}_{\text {clad }}>\mathrm{n}_{\text {ext. }}$. The angle $\theta$ gives us the numerical aperture(NA):

$$
N A=\sin (\theta)=\left(n_{\text {core }}^{2}-n_{\text {clad }}^{2}\right)^{1 / 2} .
$$

Angle $\alpha$ is the maximum angle for a ray undergoing total internal reflection at the cladding-externalmedium boundary. The cladding thickness is $\mathrm{d}$. During tapering, the fiber becomes thinner and higher-order modes are forced into the cladding layer and bounded by the cladding-external-medium interface. ${ }^{15}$ Hence the total throughput power has two parts,

$$
\mathrm{P}=\mathrm{P}_{\text {core }}+\mathrm{P}_{\text {clad }},
$$

where $\mathrm{P}_{\text {core }}$ is the power bounded by the core-cladding interface and $\mathrm{P}_{\text {clad }}$ is the cladding power bounded by the dadding-external-mediuminterface. Theshaded areas in Fig. 1 include both guided core and cladding power.

\section{A. Power Loss of the Guided Cladding Modes}

F or given $n_{\text {core }}$ and $n_{\text {clad }}, P_{\text {clad }}$ depends on the surrounding material $\left(n_{\text {ext }}\right)$. The cladding-mode power loss can be depicted by the angle $\alpha$ shown in Fig. 1. Mathematically,

$$
\alpha=\sin ^{-1}\left(\frac{n_{\text {clad }}}{n_{\text {core }}}\right)-\sin ^{-1}\left(\frac{n_{\text {ext }}}{n_{\text {core }}}\right) .
$$

The assumption that $\mathrm{n}_{\text {ext }}<\mathrm{n}_{\text {clad }}<\mathrm{n}_{\text {core, }} \mathrm{n}_{\mathrm{ext}}=1.0$ corresponds to the minimum loss and $n_{\text {ext }}=n_{\text {clad }}$ corresponds to the complete loss of the cladding modes. In the region $1.0<\mathrm{n}_{\mathrm{ext}}<\mathrm{n}_{\mathrm{clad}}$, the power loss of the cladding modes may be expressed in reference to the loss of $n_{\text {ext }}=1.0$. The result is given in Fig. 2.

\section{B. Power Loss of the Guided Core Modes}

If $\mathrm{n}_{\text {ext }}<\mathrm{n}_{\text {core }}, \mathrm{P}_{\text {core }}$ is insensitive to the external medium. However, if $n_{\text {ext }}$ is very close (nearly equal) to $\mathrm{n}_{\text {core }}$ and the cladding thickness $(\mathrm{d})$ is small (a few wavelengths), $P_{\text {core }}$ would decrease dramatically, and the core field would penetrate into the external medium because of frustrated total internal reflection (FTIR).

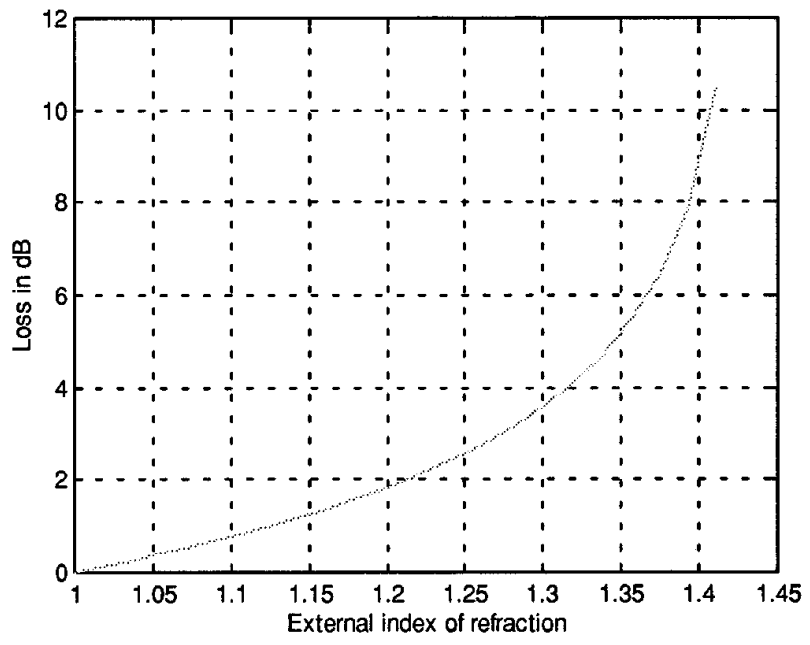

Fig. 2. Dependence of the cladding-power loss on the refractive index of the surrounding material.

Mathematically, the power loss through FTIR may be expressed by the transmission coefficient of a core-cladding-core structure, ${ }^{16}$

$$
T=1-\left|\frac{Z_{2}^{2}-Z_{1}^{2}}{Z_{1}^{2}+Z_{2}^{2}+2 i Z_{1} Z_{2} \cot \left(k_{2 z} d\right)}\right|^{2},
$$

where

$$
\begin{aligned}
& \mathrm{z}_{1}=\frac{1}{\mathrm{n}_{\text {core }} \cos (\gamma)}, \\
& \mathrm{z}_{2}=\frac{1}{\mathrm{n}_{\mathrm{clad}} \cos \left(\gamma^{\prime}\right)},
\end{aligned}
$$

for the component whose polarization direction is perpendicular to the plane of incidence, and

$$
\begin{aligned}
& \mathrm{Z}_{1}=\frac{\cos (\gamma)}{\mathrm{n}_{\text {core }}}, \\
& \mathrm{Z}_{2}=\frac{\cos \left(\gamma^{\prime}\right)}{\mathrm{n}_{\mathrm{clad}}},
\end{aligned}
$$

for the component whose polarization direction is parallel to the plane of incidence. Also

$$
\mathrm{k}_{2 \mathrm{z}}=\frac{2 \pi}{\lambda} \cos \left(\gamma^{\prime}\right),
$$

where $\lambda$ is the wavelength and $\gamma^{\prime}$ is the refractive angle in the cladding region. Because all rays bounded in the core region undergo total internal reflection at the core-cladding boundary, the angle $\gamma^{\prime}$ is a complex angle given by

$$
\cos \left(\gamma^{\prime}\right)=\mathrm{i}\left[\frac{\mathrm{n}_{\text {core }}{ }^{2}}{n_{\text {clad }}{ }^{2}} \sin ^{2}(\gamma)-1\right]^{1 / 2} .
$$

The transmission coefficient expression Eq. (5) is a function of the cladding thickness $d$. To calculate 
this thickness dependence, we assume that $\lambda=633$ $\mathrm{nm}, \mathrm{n}_{\text {core }}=1.458$, and $\mathrm{n}_{\text {clad }}=1.429(\mathrm{NA}=0.29)$. The range of the incident angle $\gamma$ is

$$
\sin ^{-1}\left(\frac{\mathrm{n}_{\text {clad }}}{n_{\text {core }}}\right) \leq \gamma \leq \frac{\pi}{2} .
$$

To simulate waveguide performance, 100 rays whose incident angles are uniformly distributed across the above range were selected. The average transmission coefficient of the perpendicular and parallel polarization components is taken as thefinal transmission value, i.e., power loss. The numerical results are shown in Fig. 3. The cladding thickness is expressed in terms of the thickness-to-wavelength ratio.

\section{Experiments}

For the purpose of discussion, we show theexperimental setup in Fig. 4. He-Ne laser light was coupled into one of the two $100 \mu \mathrm{m} / 140 \mu \mathrm{m}$ graded-index multimode fibers manufactured by SpecTran. The indices of the core and cladding are 1.458 and 1.429, respectively. The ports of the coupler are labeled 1 , 2,3 , and 4; the excitation port was port \#1. The reason for using a $\mathrm{He}-\mathrm{Ne}$ laser source is that the output-light-intensity pattern can be observed and the launch conditions may be easily adjusted and monitored to permit the excitation of lower-order modes and ensure that the far-field speckle pattern is uniform. Because the performance of multimode fiber couplers is essentially wavelength independent, 6,9 the outcomes of this study can be considered as general results.

Two technical terms, excess loss and percentage power, are frequently used in this paper and are defined bel ow:

$$
\begin{array}{r}
\text { excess loss }(\mathrm{dB})=-10 \times \log _{10}\left(\frac{\mathrm{P}_{3}+\mathrm{P}_{4}}{\mathrm{P}_{1}}\right), \\
\text { percentage power }\left(\text { port \#4) }=\frac{\mathrm{P}_{4}}{\mathrm{P}_{3}+\mathrm{P}_{4}} \times 100 \%,\right.
\end{array}
$$

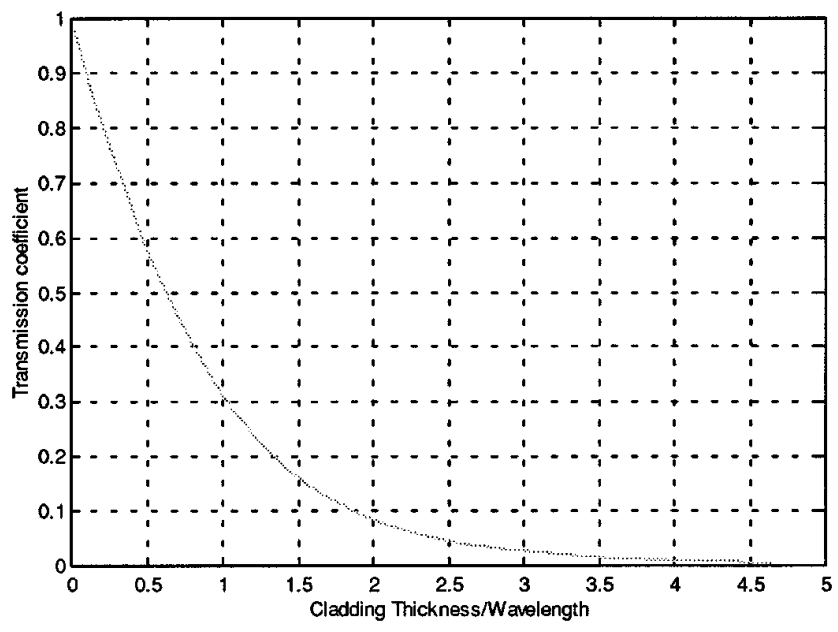

Fig. 3. Transmission coefficient (power loss) because of FTIR.

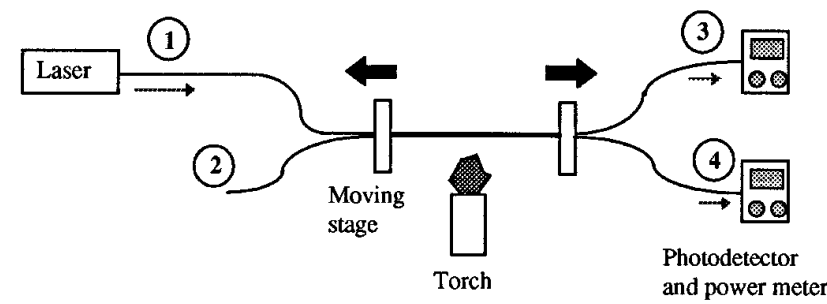

Fig. 4. Experimental setup.

where $P_{1}, P_{3}$, and $P_{4}$ represent the power in ports $\# 1$, $\# 3$, and \#4, respectively.

\section{A. External-Index Dependence for a 3-dB Coupler}

The fibers were fused and stretched until the outputpower-splitting ratio approached approximately 50/50 (actually 53/47). The taper waist section, whose cross-sectional area is shown in Fig. 5, was submerged into various liquid solutions with different indices of refraction, and the corresponding excess losses were recorded. Figure 6 shows the experimental results.

We performed this experiment by carefully cleaning the fiber taper section with alcohol every time a solution of a different refractive index was applied. It is evident that the excess loss of the 3-dB multimode coupler increases exponentially as the external refractive index changes from 1.0 (air) to a value equal to or greater than the fiber cladding index.

\section{B. External-Index Dependence for a Range of Splitting Power Ratio of $100 / 0$ through 50/50}

To determine how the external index affects the excess loss of a multimode coupler at different stages in a tapering process, alcohol (refractive index $=1.36$ ) was applied to the taper waist section, and the output

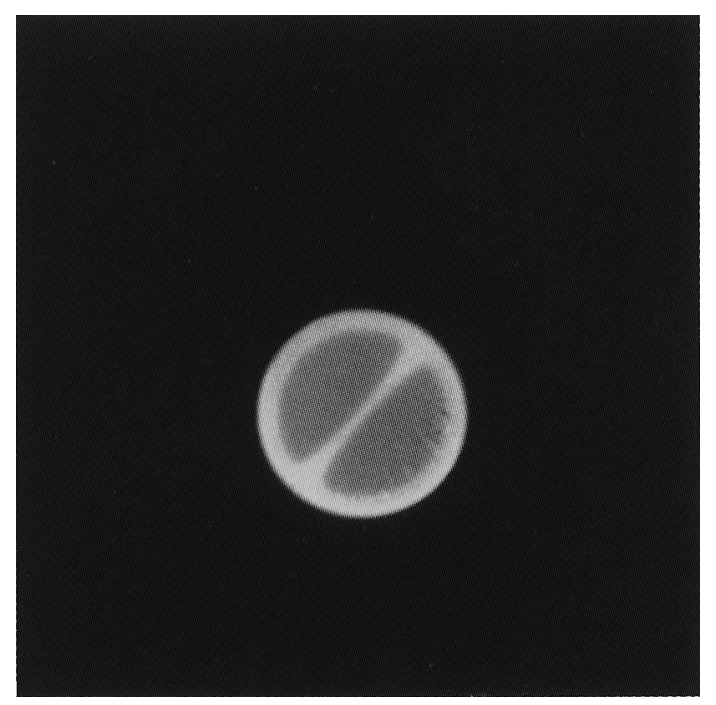

Fig. 5. Cross-sectional area of the multimode coupler taper waist. The diameter of the taper is $47 \mu \mathrm{m}$. The cladding thickness is $5.1 \mu \mathrm{m}$ and the thickness of layer between the two cores is approximately $2.5 \mu \mathrm{m}$. 


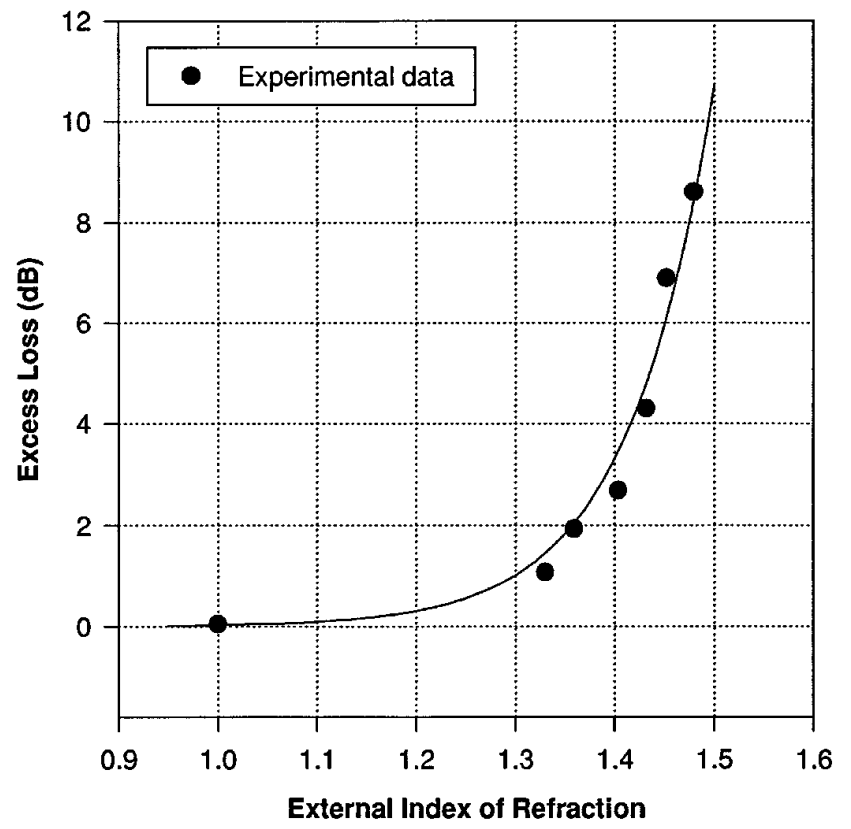

Fig. 6. Excess loss as a function of external refractive index (at $47 \%$ power in port \#4).

powers $\mathrm{P}_{3}$ and $\mathrm{P}_{4}$ for both air and alcohol as the surrounding materials were recorded. Alcohol was chosen in this experiment because it could be easily vaporized, and the tapering process could go on until the intended splitting ratio was reached. Figure 7 shows the experimental data. The excess loss is less than $0.2 \mathrm{~dB}$ before the percentage power coupled into the secondary fiber reaches nearly $40 \%$, regardless of whether air or alcohol is used as the surrounding material. Tapering beyond this point, the coupler

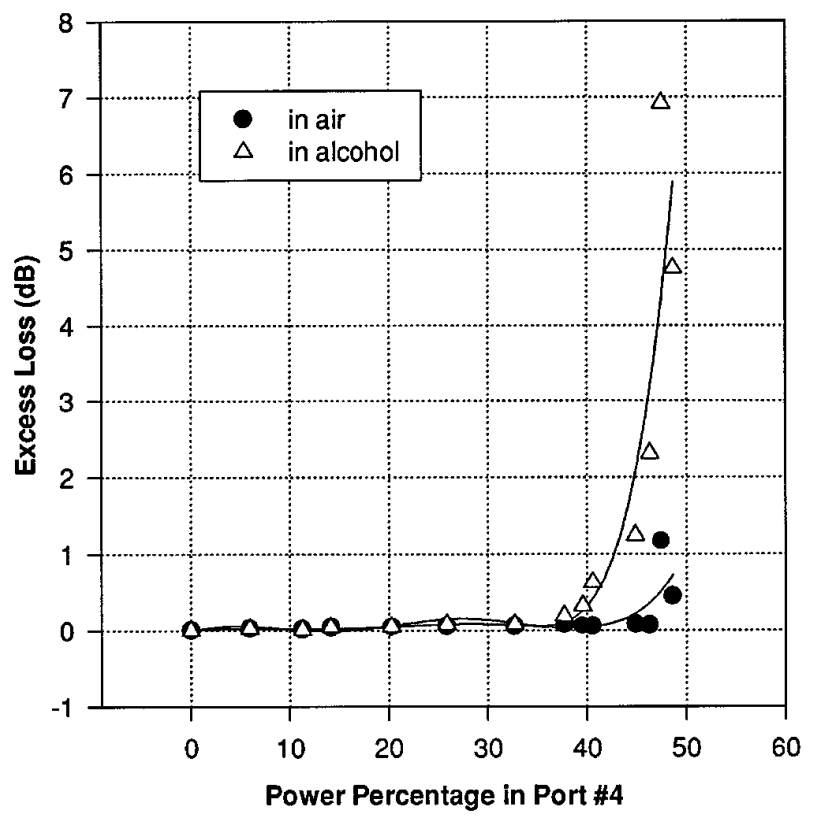

Fig. 7. Excess loss as a function of power percentage in port \#4 for different external indices of refraction. excess loss increases dramatically and hence becomes external index dependent.

\section{Conclusions}

We have studied experimentally the effect of the external index of refraction on a $2 \times 2$ multimodefiber coupler by changing the surrounding material at different steps during the tapering process. It has been identified that at the initial tapering stage (up to $40 \%$ power percentage in the secondary fiber), coupler power loss is insensitive to the surrounding material. When the coupler region is stretched beyond this point, the power loss increases as the external medium changes from air to another index-matching material with a higher index of refraction. The loss increases exponentially when the external index approaches the fiber core index, and the cladding-layer thickness becomes a few wavelengths.

Theoretically, we devel oped a ray-optics model that recognizes that the total guided power in the coupler waist region consists of the bounded core power and the trapped cladding power. The decrease of the latter is obvious as the external index increases from 1.0 to that of the cladding index, in which case the cladding rays are completely unguided. Theexternalindex dependence of the former may be depicted by the FTIR phenomenon when the external index of refraction reaches nearly that of the core, and the cladding-layer thickness is no more than a few wavelengths. Computer simulation has been performed, and the results agree with the experimental findings.

One direct application of the effect of the external index on multimode-fiber-coupler performance is in coupler packaging. Unlike a 3-dB single-mode coupler where, for a short taper length, the power loss is very small (less than $0.1 \mathrm{~dB}$ ) and independent of the surrounding material, the performance of a multimode coupler with a 50/50 splitting ratio may be sensitive to the external index. Thus directly potting epoxy onto the taper region to enhance the mechanical strength as well as to isolate the fiber taper from the external environment may affect coupler outputs. However, for a multimode fiber coupler with splitting-power ratios other than $50 / 50$, it is possible that its performance is not affected by the external index of refraction.

The authors would like to thank M. F. Gunther and R. Crotts for useful discussions regarding coupler fabrication. This research work was sponsored in part by the Virginia Center for I nnovative Technology.

\section{References}

1. M. K. Barnoski and H. R. Friedrich, "Fabrication of an access coupler with single-strand multimode fiber waveguides,"Appl. Opt. 15, 2629-2630 (1976).

2. B. S. Kawasaki and K. O. Hill, "Low-loss access coupler for multimode optical fiber distribution networks," Appl. Opt. 16, 1794-1795 (1977).

3. D. C. J ohnson, B. S. Kawasaki, and K. O. Hill, "F used biconical tapered fiber optic devices: application to data buses," Fiber Integrated Opt. 3, 263-284 (1980). 
4. V. J . Tekippe, "Passive fiber-optic components made by the fused biconical taper process," Fiber I ntegrated Opt. 9, 97-123 (1990).

5. A. W. Snyder and P. Mcl ntyre, "Crosstalk between light pipes," J . Opt. Soc. Am. 66, 877-882 (1976).

6. K. Ogawa, "Simplified theory of the multimode fiber coupler," Bell Syst. Tech. J . 56, 729-745 (1977).

7. F. Szarka, A. Lightstone, J . Lit, and R. Hughes, "A review of biconical taper couplers," Fiber Integrated Opt. 3, 285-298 (1980).

8. Y-F. Li and J. W. Y. Lit, "Coupling efficiency of a multimode biconical taper coupler,"J . Opt. Soc. Am. A 2, 1301-1306 (1985).

9. R. Griffin, J. D. Love, P. R. A. Lyons, D. A. Thorncraft, and S. C. Rashleigh, "Asymmetric multimode couplers," J . Lightwave Technol. 9, 1508-1517 (1991).

10. R. G. Lamont, D. C. J ohnson, and K. O. Hill, "Power transfer in fused biconical-taper single mode fiber couplers: depen- dence on external refractive index," Appl. Opt. 24, 327-332 (1985).

11. W. K. Burns, M. Abebe, C. A. Villarruel, and R. P. Moeller, "Loss mechanisms in single-mode fiber tapers," J . Lightwave Technol. 4, 608-613 (1986).

12. F. P. Payne, T. Finegan, M. S. Yataki, R. J . Mears, and C. D. Hussey, "Dependence of fused taper couplers on external refractive index,"Electron. Lett. 22, 1207-1209 (1986).

13. A. Fielding and T. Bricheno, "Single mode fused couplers insensitive to external refractive index," Electron. Lett. 24, 851-853 (1988).

14. X. H. Zheng and A. W. Snyder, "F used couplers: condition for insensitivity to external refractive index," Electron. Lett. 23, 182-184 (1987).

15. D. Marcuse, Theory of Dielectric Optical Waveguides, 2nd ed. (Academic, San Diego, Calif., 1991), Chaps. 1 and 2.

16. L. M. Brekhovskiskh, Waves in Layered Media, 2nd ed. (Academic, San Diego, Calif., 1980), Chap. 1. 